Original paper

\title{
Dosimetric response of a glass dosimeter in proton beams: LET-dependence and correction factor
}

\author{
Keisuke Yasui $^{\text {a, }}{ }^{*}$, Chihiro Omachi ${ }^{\mathrm{b}}$, Junya Nagata ${ }^{\mathrm{c}}$, Toshiyuki Toshito ${ }^{\mathrm{b}}$, Hidetoshi Shimizu ${ }^{\mathrm{d}}$, \\ Takahiro Aoyama ${ }^{\mathrm{d}}$, Naoki Hayashi ${ }^{\mathrm{a}}$ \\ ${ }^{a}$ Fujita Health University, Faculty of Radiological Technology, School of Health Sciences, Japan \\ ${ }^{\mathrm{b}}$ Nagoya Proton Therapy Center, Nagoya City West Medical Center, Japan \\ ${ }^{\mathrm{c}}$ Graduate School of Health Sciences, Fujita Health University, Japan \\ d Department of Radiation Oncology, Aichi Cancer Center Hospital, Japan
}

\section{A R T I C L E I N F O}

\section{Keywords:}

Radiophotoluminescent glass dosimeter

Linear energy transfer

Proton beam

Under-response correction

Postal audit

\begin{abstract}
A B S T R A C T
A radiophotoluminescent glass dosimeter (RGD) is widely used in postal audit system for photon beams in Japan. However, proton dosimetry in RGDs is scarcely used owing to a lack of clarity in their response to beam quality. In this study, we investigated RGD response to beam quality for establishing a suitable linear energy transfer (LET)-corrected dosimetry protocol in a therapeutic proton beam.

The RGD response was compared with ionization chamber measurement for a $100-225 \mathrm{MeV}$ passive proton beam. LET of the measurement points was calculated by the Monte Carlo method. An LET-correction factor, defined as a ratio between the non-corrected RGD dose and ionization chamber dose, of $1.226 \times(\text { LET })^{-0.171}$ was derived for the RGD response. The magnitude of the LET-dependence of RGD increased with LET; for an LET of $8.2 \mathrm{keV} / \mu \mathrm{m}$, the RGD under-response was up to $16 \%$. The coefficient of determination, mean difference \pm SD of non-corrected RGD dose, residual range-corrected RGD dose, and LET-corrected RGD dose to the ionization chamber are $0.923,3.7 \pm 4.2 \%,-2.4 \pm 7.5 \%$, and $0.04 \pm 2.1 \%$, respectively. The LET-corrected RGD dose was within $5 \%$ of the corresponding ionization chamber dose at all energies until $200 \mathrm{MeV}$, where it was $5.3 \%$ lower than the ionization chamber dose.

A corrected LET-dependence of RGD using a correction factor based on a power function of LET and precise dosimetric verification close to the maximum LET were realized here. We further confirmed establishment of an accurate postal audit under various irradiation conditions.
\end{abstract}

\section{Introduction}

In recent years, both active scanning proton beam and passive proton beam have been widely used in the treatment of various types of tumors $[1,2]$. Several physical characteristics of a proton beam, such as a beam energy-dependent Bragg peak and a beam range, render dosimetric advantages to proton beams over the conventional photon therapy. Similar to the implementation of photon therapy, accurate commissioning, dosimetric verification of the treatment planning system (TPS), and quality assurance are fundamental for implementing high-quality proton therapy [3-5]. To verify the proton dose, various detectors, such as an ionization chamber, a radiochromic film, a metal oxide-semiconductor field-effect transistor (MOSFET) detector, a thermoluminescent dosimeter (TLD), an optically stimulated luminescence dosimeter (OSLD), a diamond detector, and a radiophotoluminescent glass dosimeter (RGD), have been investigated and used [6-10]. Radiochromic films exhibit a decreasing linear response to increasing linear energy transfer (LET) [6]. According to the study, EBT3 film showed an under-response of approximately $20 \%$ at an LET of $9.27 \mathrm{keV} / \mu \mathrm{m}$ near the Bragg peak; the absolute dose difference was within $2 \%$ by using a linear LET-correction factor. Similar trend was observed for a MOSFET dosimeter with a maximum dose difference of $26 \%$ as the MOSFET dosimeter properly worked after the application of an LET correction according to the measurement depth [7]. TLDs are reported to be mostly stable for small changes in LET and have a dose accuracy of less than $5 \%$ [11]. Moreover, TLDs and OSLs exhibit an over-response and an underresponse of approximately $7 \%$ and $4 \%$, respectively, except in the distal region $[8,12]$. TLDs have been used as a point dosimetry tool in the

\footnotetext{
* Corresponding author.

E-mail address: k-yasui@fujita-hu.ac.jp (K. Yasui).
} 
Imaging and Radiation Oncology Core cooperative's proton beam clinical trial credentialing with a dose criterion in the range of 5-7\% [13]. For the diamond detector, no LET dependence has been observed for low-energy protons [14]. LET dependence has also not been observed for the clinically used high-energy protons; however, there is a nonreproducibility among the devices $[10,15]$.

RGDs were used in the postal dose audit of photon beams in Japan [16-18]. An RGD exhibits excellent dosimetric characteristics such as uniformity, reproducibility, good dose linearity, and energy dependency, and the possibility of in vivo measurements using an RGD with proton and photon beams has been reported $[9,19]$. In addition, an RGD is useful for postal audits of proton beams with residual range $\left(R_{\text {res }}\right)$ correction $[20,21]$. In our previous work, we investigated the correction of the stopping power ratio and LET quenching for RGD dosimetry in a passive $200 \mathrm{MeV}$ proton beam [20]. We showed that the combined uncertainty of RGD dosimetry using $\mathrm{R}_{\text {res }}$-based correction was approximately $3.6 \%$, and an RGD was feasible as a postal audit dosimeter for proton beam therapy where $R_{\text {res }}>1 \mathrm{~cm}$. However, the RGD response for various proton energies used in clinical settings was not investigated except for a $200 \mathrm{MeV}$ proton beam. Since the LET is also affected by the spread-out Bragg peak (SOBP) width, $\mathrm{R}_{\text {res-based correction may not }}$ provide sufficiently accurate dosimetry under various clinical conditions. Araki et al. studied the response of an RGD to various photon and electron beam energies in detail, and for practical use of the RGD, they recommended either a direct measurement of the RGD response or the use of the Monte Carlo (MC) simulated RGD response in the beam quality relative to the reference source [22]. Besides, the postal audit of non-reference conditions, such as the wedge field and IMRT, have been studied for photon therapy $[17,18]$. However, the response of RGDs under various conditions for proton therapy has to be investigated. The purpose of this study is to investigate the RGD response for therapeutic proton beams to establish a suitable dosimetry protocol considering the correction for LET using MC simulations. Determining the LET

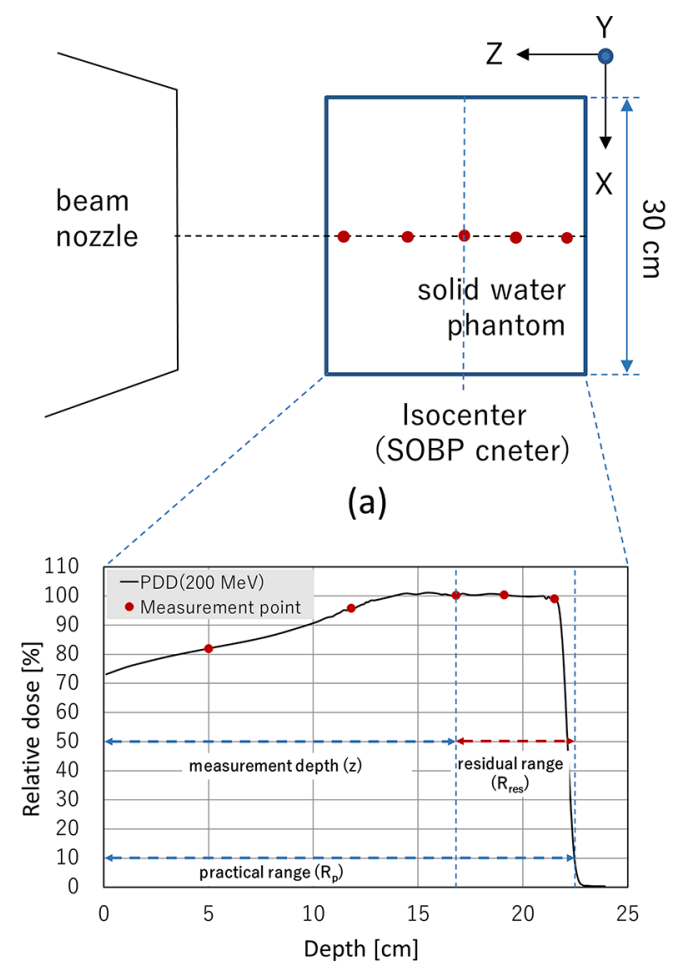

(b) dependence of RGDs enables audits to be performed to assure doses for various proton treatments, such as IMPT.

\section{Materials and methods}

\subsection{Beam delivery system}

The beam delivery system used in this study was PROBEAT-III (Hitachi Ltd., Japan) installed at Nagoya Proton Therapy Center (NPTC). NPTC has three treatment rooms for spot scanning and two passive scattering rooms with a range modulation wheel (RMW) [23]. The accelerator at NPTC produces proton beams with eight different energies in the range from 100 to $250 \mathrm{MeV}$ for the passive method. We performed this study using passive proton beams with seven different energies in the range of 100-225 MeV. In passive scattering systems at NPTC, high-energy passive beams are mainly used in the prostate cancer treatment, [24,25] and middle- or low-energy passive beams are primarily used with respiratory gating in the treatment of the lung and liver tumors $[26,27]$. By RMW rotation in combination with the beam gating, each RMW can produce SOBPs in the range from 1 to $16 \mathrm{~g} / \mathrm{cm}^{2}$ at an interval of $1 \mathrm{~g} / \mathrm{cm}^{2}$ according to the range. The reference SOBP widths for protons with high energy $(180,200$, and $225 \mathrm{MeV})$, intermediate energy (140 and $160 \mathrm{MeV}$ ), and low energy (100 and $120 \mathrm{MeV}$ ) were set at $10 \mathrm{~cm}, 6 \mathrm{~cm}$, and $3 \mathrm{~cm}$, respectively, depending on their ranges. The field size was $10 \times 10 \mathrm{~cm}^{2}$ at the isocenter, which is the SOBP center of each energy. In this study, LET was calculated, and RGD responses were verified at these reference SOBP widths.

\subsection{Dosimetry and LET correction of the RGD}

RGDs (GD-302M, Asahi Techno Glass, Japan) and an automatic reader (FGD-1000, Asahi Techno Glass, Japan) were used in this study. Fig. 1(a) and (b) show the experimental setup and a curve correlating the

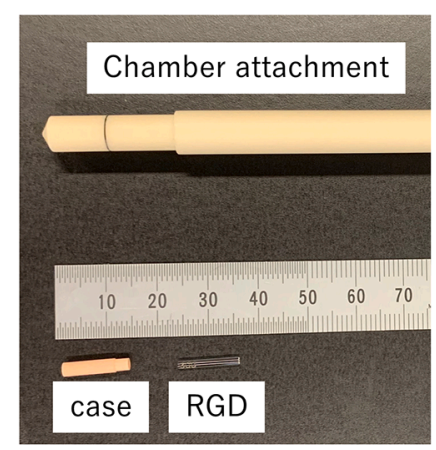

(c)

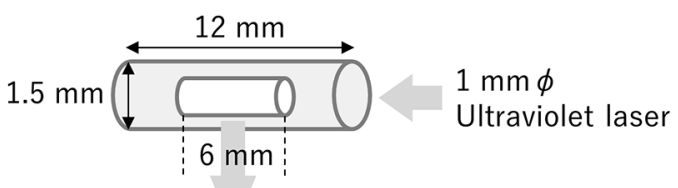

radiophotoluminescent

Dimension of RGD: $\phi 1.5 \times 12 \mathrm{~mm}$ Readout volume: $\phi 1.0 \times 6 \mathrm{~mm}$

(d)

Fig. 1. (a) Experimental setup and (b) a correlation between the measurement points and percentage depth dose (PDD). The red dots show the measurement points. (c) Schematic figure of RGD and chamber attachment for measurement and (d) schematic figure showing the magnification of the RGD and readout volume. (For interpretation of the references to colour in this figure legend, the reader is referred to the web version of this article.) 
measurement points and PDD, respectively. Schematic figures of an RGD and chamber attachment are illustrated in Fig. 1(c), and (d) displays the readout volume. A model GD-302M RGD has a diameter and length of $1.5 \mathrm{~mm}$ and $12 \mathrm{~mm}$, respectively. The readout volume has $1 \mathrm{~mm}$ diameter and $6 \mathrm{~mm}$ length, and it is located opposite to the serial number. The variation of reproducibility for the reading of the RGD is less than $0.9 \%$ for photon beams above $20 \mathrm{keV}[16,28]$. All RGDs were annealed at $400{ }^{\circ} \mathrm{C}$ for $1 \mathrm{~h}$ before measurement. Further, to improve the fluorescence stability, readings were taken after preheating at $70{ }^{\circ} \mathrm{C}$ for $0.5 \mathrm{~h}$. RGDs exhibit excellent dosimetric characteristics in terms of uniformity and reproducibility $( \pm 1.2 \%)$, good dose linearity ranging up to $30 \mathrm{~Gy}$, and energy dependency, which is within $2 \%$ for photon beams [29-31]. Moreover, they are less prone to fading and can be read repeatedly. We used a $200 \mathrm{MeV} / \mathrm{SOBP} 10 \mathrm{~cm}$ beam, which is the reference condition at NPTC, to calibrate the RGDs. The absolute dose to water was determined using a PinPoint 3D Ion Chamber (model 31016, PTW, Germany) with a sensitivity volume of $1.45 \mathrm{~mm}$ radius and 2.6 $\mathrm{mm}$ length. At each measurement point, we used the RGD with a chamber attachment as well as the ionization chamber. $R_{\text {res }}$ of each point was defined as $R_{\text {res }}=\left(R_{p}-z\right)$, where $R_{p}$ is the practical range defined as the depth at which the dose falls to $10 \%$ of the dose at the SOBP center [32], and $\mathrm{z}$ is the measurement depth. The measurement depths were estimated at the following positions: center of SOBP, 2-4 mm inside the distal end of the range, between the SOBP center and distal point, the proximal side of the SOBP, and $5 \mathrm{~cm}$ depth.

According to our previous study, a quenching effect correction is required for an accurate RGD dose measurement, and the quenching correction factors are associated with $\mathrm{R}_{\text {res }}[20]$. $\mathrm{R}_{\text {res }}$ indicates the proton energy [33] and is a recommended beam quality index in the proton dosimetry protocol TRS-398 and standard dosimetry in Japan [32,34]. The $R_{\text {res }}$ correction equation presented in the previous work is summarized as follows:

$k_{Q, Q_{0}}^{R G D}\left(R_{\text {res }}\right)=0.994+0.052 e^{-0.537 R_{\text {res }}}$ and

$k_{L E T}^{R G D}\left(R_{\text {res }}\right)=\frac{1}{0.521+0.476 e^{-0.538\left(R_{\text {res }}\right)^{-0.4}}}$,

where $k_{Q, Q_{0}}^{R G D}\left(R_{r e s}\right) \operatorname{and} k_{L E T}^{R G D}\left(R_{r e s}\right)$ are the correction factors for the beam quality and the LET quenching effect, respectively. $R_{\text {res-corrected RGD }}$ dose (Rres-correction) was calculated by multiplying the calibrated dose under the reference condition by both beam quality (Eq. 1) and LET (Eq. 2) correction factors. However, as mentioned above, since LET is affected by irradiation conditions such as energy and SOBP width, it is difficult to indicate LET using $\mathrm{R}_{\text {res }}$ in low-energy proton beams.

In this study, we compared the LET-corrected RGD dose (LETcorrection) with Rres-correction to investigate the response characteristics of RGDs for therapeutic proton beams. We performed an MC simulation to calculate LET and measured the quenching effect of an RGD at several energies and SOBPs of therapeutic proton beams at multiple depths. The LET-dependence of RGD was quantified as a ratio between the non-corrected RGD dose and the ionization chamber dose. An RGD response-correction factor, which is a function of LET, was derived. LET values approximately ranged from 1.5 to $8.0 \mathrm{keV} / \mu \mathrm{m}$. Irradiated doses were $200 \mathrm{MU}$ for each energy. The LET of the reference point where the RGD was calibrated was $3.27 \mathrm{keV} / \mu \mathrm{m}$. We measured the LET-dependence of RGD and estimated the power function from data fitting. The LET-correction was then calculated by multiplying the uncorrected RGD dose (u-RGD) by the obtained function.

\subsection{Monte Carlo simulation}

The Geant4 version 10.2 MC toolkit [35,36] was used to calculate LET in water for each energy and SOBP. The component modules of the proton therapy system reproduced in Geant 4 were all the modules in the beamline including RMW, second scatter, and main/sub dose monitor.
The RMWs of NPTC have six modulation regions per rotation. In the MC calculation, gating patterns to create SOBPs were tuned to provide simulation results that agreed well with the measured SOBPs.

Moreover, we reproduced the materials and shape of the second scatter. The MC calculation was performed with a virtual water phantom, and the detection geometry of the LET was fixed at $12 \times 3 \times 1.5$ $\mathrm{mm}^{3}$. The $12 \mathrm{~mm}$ length and $1.5 \mathrm{~mm}$ thickness correspond to the actual RGD dimensions. However, to account for the statistical error, the lateral dimension is taken to be wider than the actual corresponding RGD dimension as it is less sensitive to changes in LET. Although there are various methods for calculating LET, we followed previous studies and used the dose-averaged LET $[6,20]$. The LET is given as

$\operatorname{LET}(z)=\frac{\int \Phi_{E}(z) L E T^{2}(E) d E}{\int \Phi_{E}(z) L E T(E) d E}$

where $\Phi_{E}(\boldsymbol{z})$ is the fluence of protons with an energy $\mathrm{E}$ at a depth z. LET (E) is the LET with the energy E. We simulated all kinds of particles, including protons, electrons, photons, ions and neutrons. We used physics model G4HadronPhysicsFTF_BIC for hadronic interaction and G4EmStandardPhysics_option3 for electromagnetic interaction. The statistical uncertainties in the LET calculation were within $5 \%$.

\section{Results}

\subsection{Correlation of LET and $R_{\text {res }}$ for each energy}

LET as a function of $R_{\text {res }}$ of a therapeutic proton beam using MC simulation is shown in Fig. 2. For all the energies, we observe a rapid increase in LET for $R_{\text {res }}$ values below $2 \mathrm{~cm}$. Even at same $R_{\text {res, }}$ there is a difference in LET in the range of 10-30\% depending on the energy/SOBP width.

\subsection{LET-dependence of RGD}

Fig. 3 illustrates the variation of the LET-correction factor of RGD with LET. The LET dependence increases with LET, and the RGD underresponse is up to $16 \%$ at high LETs near the end of beam range. The dotted line is a correction curve for the LET fitted by a power function that describes the relationship between the RGD response and LET. The LET-dependence of RGD as a function of LET is LETcorrectionfactor = $1.226 \times(\text { LET })^{-0.171}$. The value of the coefficient of determination (R2) was 0.923 . The vertical error bars shown in Fig. 3 represent the measurement dose uncertainty. In the SOBP and plateau regions, the dose uncertainty is $\pm 2 \%$, and at the distal region, the dose uncertainty is $\pm 3 \%$. The horizontal error bars represent the variation of LET with the measurement depth uncertainty ( $\pm 2 \mathrm{~mm}$ ); for example, in the SOBP and plateau regions of high or intermediate energies, LET uncertainties are around $0.01 \mathrm{keV} / \mu \mathrm{m}$ approximately. However, for low energies, the LET uncertainty is as large as $0.12 \mathrm{keV} / \mu \mathrm{m}$ in the SOBP region. At the distal point, the maximum value of the LET uncertainty is $1.52 \mathrm{keV} / \mu \mathrm{m}$.

\subsection{Response of RGD to therapeutic proton beam}

Fig. 4(a-c) shows the dose difference vs. measurement depth, $\mathrm{R}_{\text {res }}$ and LET between RGD dose and chamber dose, respectively. For each symbol, the uncorrected RGD dose (u-RDG: white circle), $\mathrm{R}_{\text {res}}$-based correction RGD dose (Rres-correction: filled triangle), LET-based correction RGD dose (LET-correction: filled circles) are shown. The mean difference \pm SD of the errors in u-RGD, Rres-correction, and LETcorrection are $3.7 \pm 4.2 \%,-2.4 \pm 7.5 \%$, and $0.04 \pm 2.1 \%$, respectively. Visibly, no significant trend is observed for depth in Fig. 4(a). At the end of each energy range, the error in u-RGD is approximately $10-16 \%$ under-response. In addition, at shallow measurement depths, where the low-energy beam is dominant, large errors in u-RGD and Rres-correction are observed. In Fig. 4(b), the distal, in the SOBP, and the plateau region 


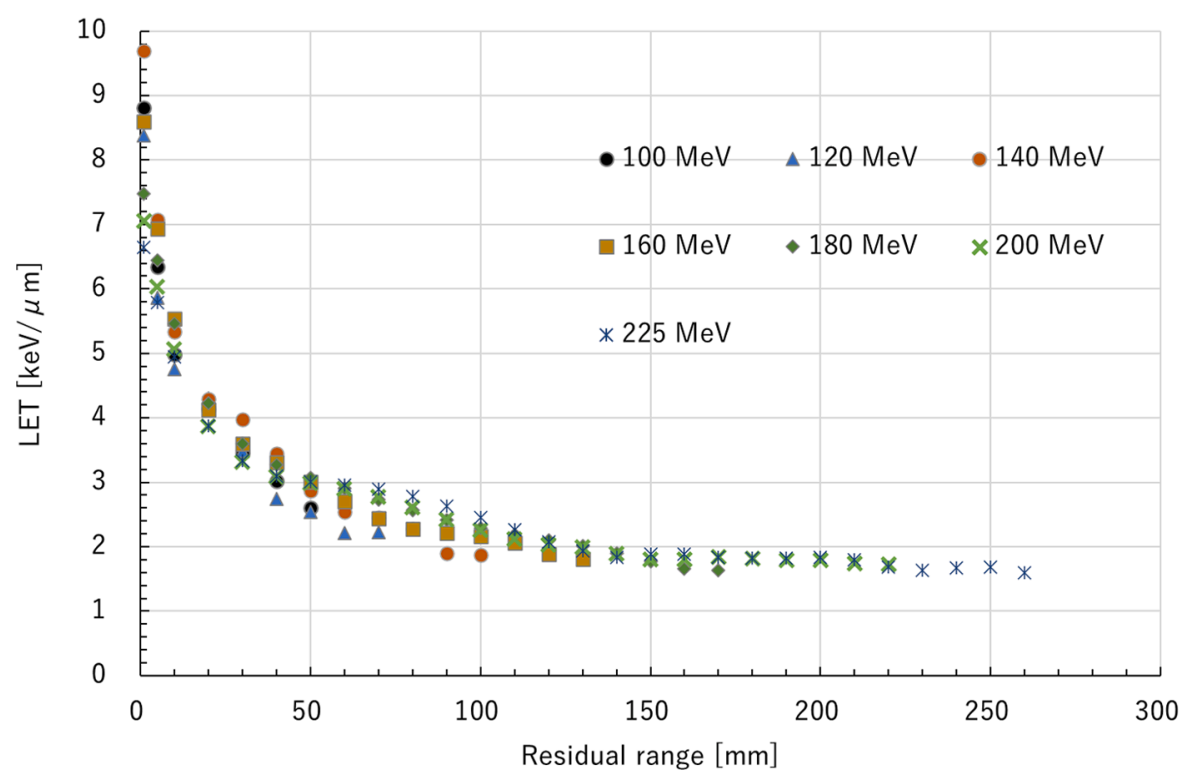

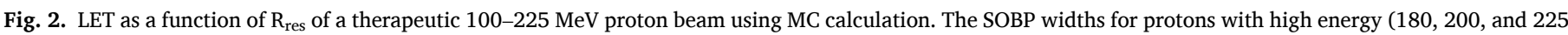
$\mathrm{MeV}$ ), intermediate energy (140 and $160 \mathrm{MeV}$ ), and low energy (100 and $120 \mathrm{MeV}$ ) are set at 10, 6, and $3 \mathrm{~cm}$, respectively.

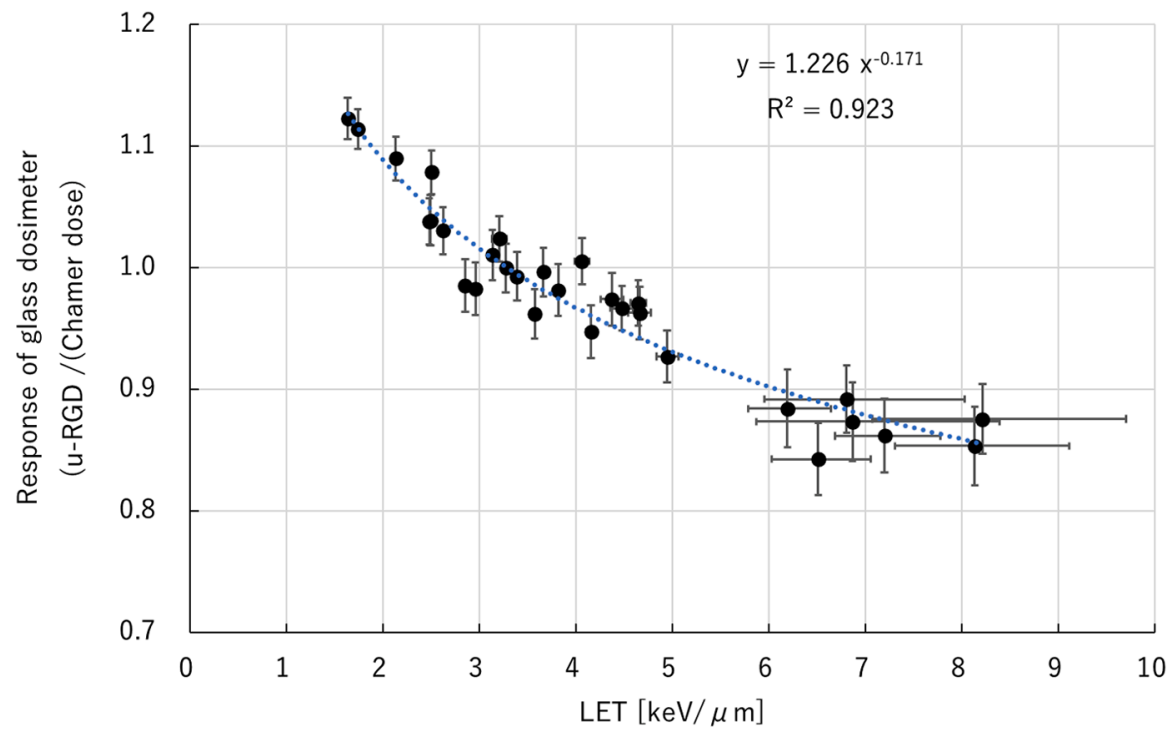

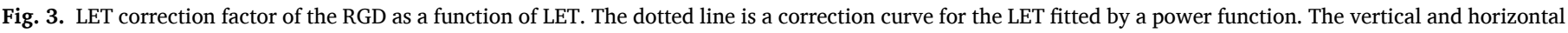
error bars show the dose uncertainty and the variation of LET with the measurement depth uncertainty, respectively.

before the SOBP are indicated; at small $\mathrm{R}_{\text {res}}$, overcorrection of Rrescorrection and the effectiveness of LET-correction are shown. In Fig. 4 (c), overcorrection in Rres-correction is observed for LET values above 4 $\mathrm{keV} / \mu \mathrm{m}$ with a maximum dose difference of $13 \%$; however, for some high LET values, the overcorrection is less than $5 \%$. Fig. 5 shows the absolute depth doses measured using the ionization chamber and RGD. Overcorrections in Rres-correction in the range of 8-9\% are observed for low-energy proton beams. Even for the intermediate energy SOBP center measurement, the Rres-correction is $2.8 \%$ higher than the ionization chamber measurement. However, the LET-corrections agree well with the ionization chamber measurements at all energies although a $5.3 \%$ lower LET-correction than the ionization chamber measurement at the end of the $200 \mathrm{MeV}$ is observed. The details of each measurement point are summarized in Table 1.

\section{Discussion}

In this study, for a therapeutic passive proton beam, we observed that the magnitude of the LET-dependence of RGD approached $15.7 \%$ at the distal end of SOBP. This LET-dependence is consistent with our previous work [20]. Furthermore, the LET-dependence of RGD is fitted as a power function of LET expressed as: LETcorrectionfactor $=1.226 \times$ $(\mathrm{LET})^{-0.171}$ in the ranges of $1.6-8.2 \mathrm{keV} / \mu \mathrm{m}$ and $3.0-21.9 \mathrm{~cm}$ for LET and $R_{\text {res, }}$ respectively. Rah et al. showed that the LET-dependence of RGD was fitted by a second polynomial in the range of $R_{\text {res }} 2.1-9.0 \mathrm{~cm}$ [21]. Our previous work showed that the $R_{\text {res }}$-based correction provided an accuracy of $\pm 3 \%$ for $R_{\text {res }}$ in the range from $1 \mathrm{~cm}$ to $15 \mathrm{~cm}$ [20]. Although several previous studies have proposed $R_{\text {res }}$-based correction methods, more clinical conditions, such as low-energy proton beams, require LET-based correction. Furthermore, the LET-based correction will potentially enable us to measure the distal end of the proton beam 

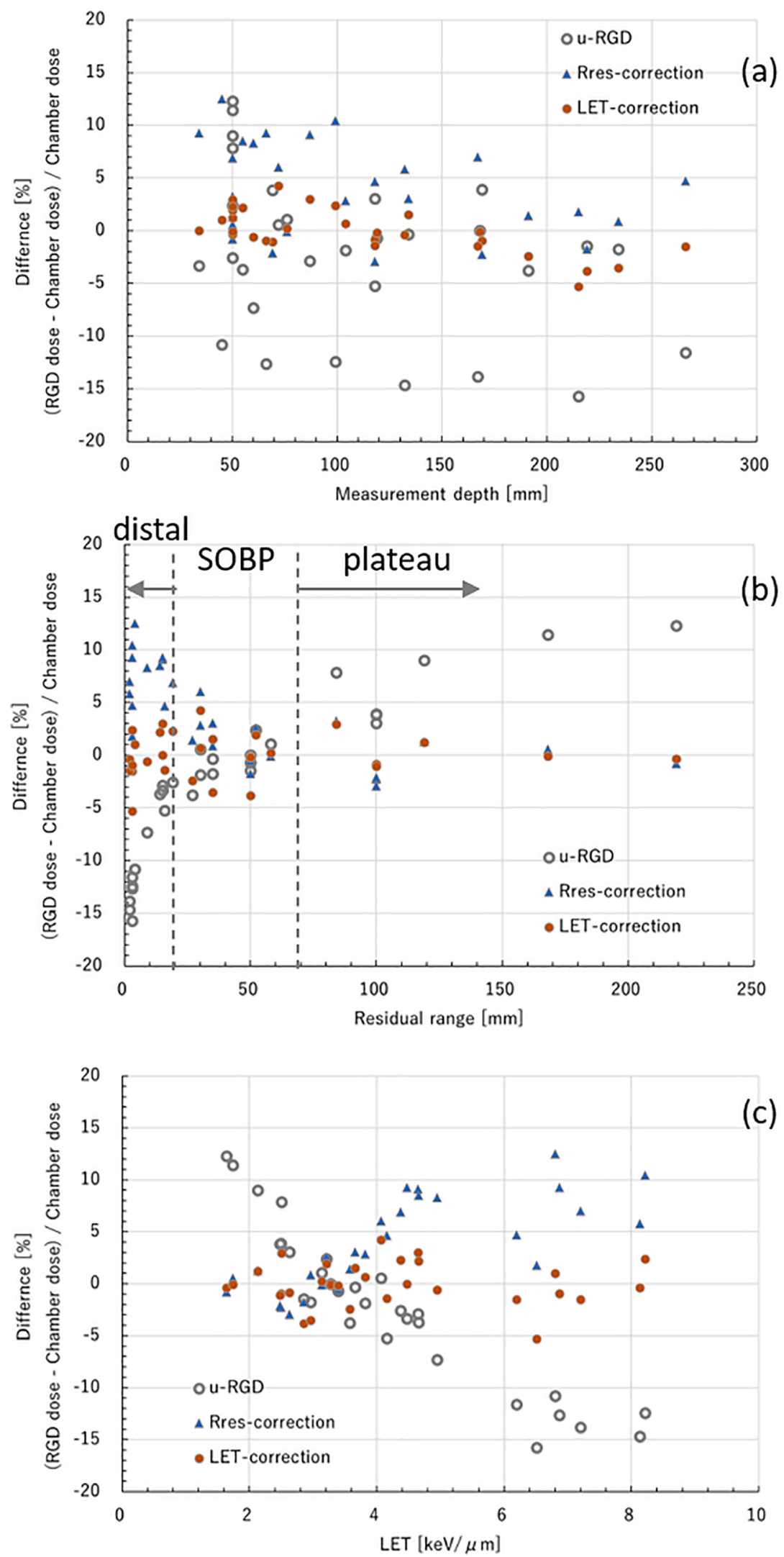

Fig. 4. Variation of dose difference between RGD dose and chamber dose with (a) measurement depth, (b) $R_{\text {res}}$, and (c) LET, respectively. $u-R G D$, Rres-correction, and LET-correction data points are represented by white circles, filled triangles, and filled circles, respectively. 


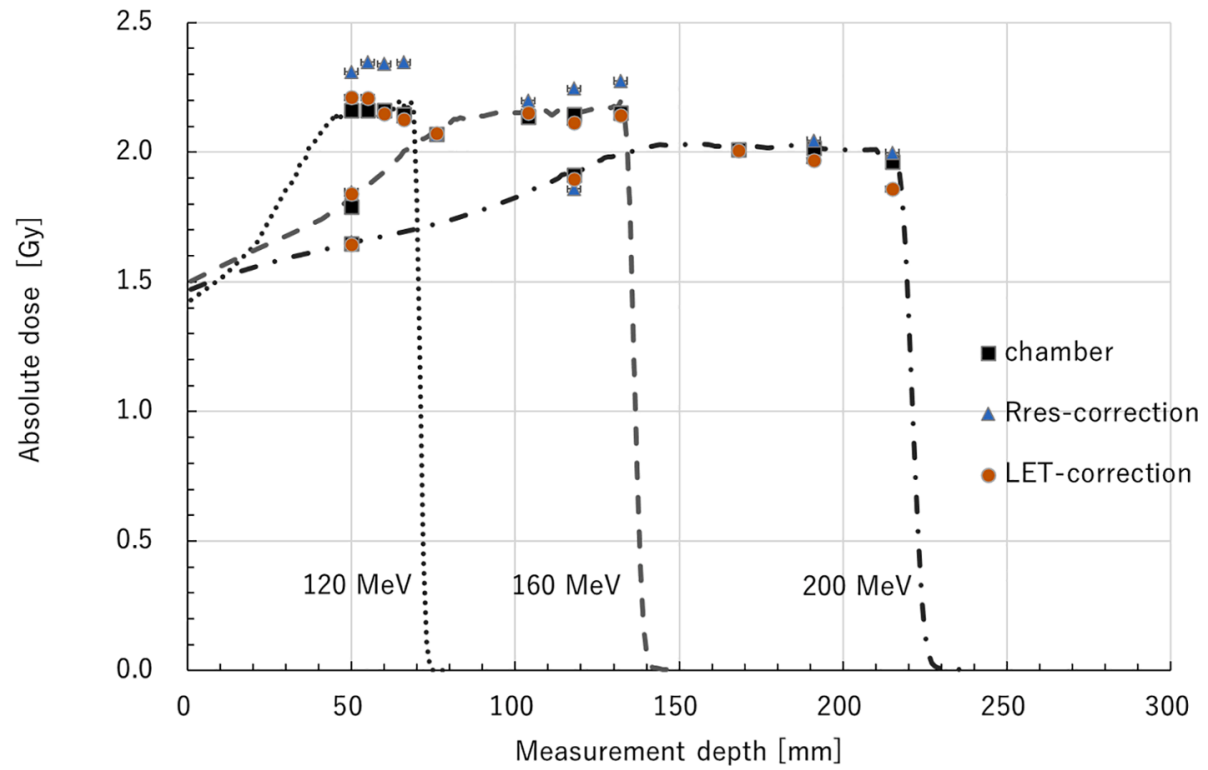

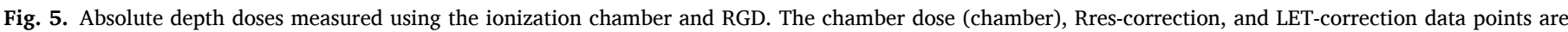

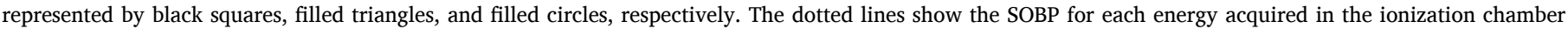
during commissioning. The horizontal error bars show the measurement depth uncertainty, which is $2 \mathrm{~mm}$.

Table 1

Details of each measurement point in terms of measurement conditions, absolute doses of ionization chambers, and differences between RGD and ionization chamber doses. (Meas.: Measurement, Uncer.: Uncertainty, Diff.: Difference, Co.: Correction).

\begin{tabular}{|c|c|c|c|c|c|c|c|c|}
\hline $\begin{array}{l}\text { Energy, Range, } \\
\text { SOBP width }\end{array}$ & $\begin{array}{l}\text { Meas. Depth } \\
(\mathrm{mm})\end{array}$ & $\begin{array}{c}\mathrm{R}_{\mathrm{res}} \\
(\mathrm{mm})\end{array}$ & $\begin{array}{c}\mathrm{LET} \\
\mathrm{keV} / \mu \mathrm{m})\end{array}$ & $\begin{array}{c}\text { LET Uncer. } \\
(\mathrm{keV} / \mu \mathrm{m})\end{array}$ & $\begin{array}{c}\text { chamber dose } \\
\text { (Gy) }\end{array}$ & $\begin{array}{l}\text { Diff. LET- } \\
\text { co. }(\%)\end{array}$ & $\begin{array}{c}\text { Diff. Rres- } \\
\text { co. }(\%)\end{array}$ & $\begin{array}{c}\text { Diff. u- } \\
\text { RGD (\%) }\end{array}$ \\
\hline $225 \mathrm{MeV}$ & 50 & 219 & 1.63 & 0.01 & 1.72 & -0.4 & -0.8 & 12.3 \\
\hline $269 \mathrm{~mm}$ & 169 & 100 & 2.49 & 0.01 & 2.06 & -0.9 & -2.3 & 3.9 \\
\hline \multirow[t]{3}{*}{$100 \mathrm{~mm}$} & 219 & 50 & 2.85 & 0.01 & 2.17 & -3.8 & -1.8 & -1.5 \\
\hline & 234 & 35 & 2.96 & 0.01 & 2.16 & -3.5 & 0.9 & -1.8 \\
\hline & 266 & 3 & 6.19 & 0.45 & 2.13 & -1.5 & 4.7 & -11.6 \\
\hline $200 \mathrm{MeV}$ & 50 & 168 & 1.74 & 0.03 & 1.65 & -0.1 & 0.6 & 11.4 \\
\hline $218 \mathrm{~mm}$ & 118 & 100 & 2.62 & 0.03 & 1.91 & -0.9 & -2.9 & 3.1 \\
\hline \multirow[t]{3}{*}{$100 \mathrm{~mm}$} & 168 & 50 & 3.27 & 0.03 & 2.01 & -0.1 & 0.0 & 0.0 \\
\hline & 191 & 27 & 3.57 & 0.03 & 2.02 & -2.4 & 1.4 & -3.8 \\
\hline & 215 & 3 & 6.51 & 0.54 & 1.96 & -5.3 & 1.8 & -15.7 \\
\hline $180 \mathrm{MeV}$ & 50 & 119 & 2.13 & 0.04 & 1.80 & 1.2 & 1.3 & 9.0 \\
\hline $169 \mathrm{~mm}$ & 69 & 100 & 2.48 & 0.04 & 1.92 & -1.1 & -2.1 & 3.8 \\
\hline \multirow{3}{*}{$100 \mathrm{~mm}$} & 119 & 50 & 3.39 & 0.04 & 2.01 & -0.2 & -0.5 & -0.7 \\
\hline & 134 & 35 & 3.66 & 0.04 & 2.00 & 1.5 & 3.1 & -0.3 \\
\hline & 167 & 2 & 7.20 & 0.58 & 2.02 & -1.5 & 7.0 & -13.8 \\
\hline $160 \mathrm{MeV}$ & 50 & 84 & 2.50 & 0.05 & 1.79 & 2.9 & 3.3 & 7.9 \\
\hline $134 \mathrm{~mm}$ & 76 & 58 & 3.13 & 0.05 & 2.07 & 0.2 & -0.1 & 1.1 \\
\hline \multirow[t]{3}{*}{$60 \mathrm{~mm}$} & 104 & 30 & 3.81 & 0.05 & 2.14 & 0.7 & 2.8 & -1.9 \\
\hline & 118 & 16 & 4.16 & 0.05 & 2.15 & -1.4 & 4.7 & -5.3 \\
\hline & 132 & 2 & 8.13 & 0.98 & 2.15 & -0.4 & 5.8 & -14.7 \\
\hline $140 \mathrm{MeV}$ & 50 & 52 & 3.21 & 0.08 & 1.88 & 1.9 & 2.6 & 2.4 \\
\hline $102 \mathrm{~mm}$ & 72 & 30 & 4.06 & 0.08 & 1.91 & 4.3 & 6.0 & 0.6 \\
\hline \multirow[t]{2}{*}{$60 \mathrm{~mm}$} & 87 & 15 & 4.65 & 0.08 & 1.89 & 3.0 & 9.1 & -2.9 \\
\hline & 99 & 3 & 8.21 & 1.49 & 1.92 & 2.4 & 10.4 & -12.4 \\
\hline $120 \mathrm{MeV}$ & 50 & 19 & 4.37 & 0.11 & 2.16 & 2.3 & 6.9 & -2.6 \\
\hline $69 \mathrm{~mm}$ & 54 & 15 & 4.66 & 0.11 & 2.16 & 2.2 & 8.5 & -3.7 \\
\hline \multirow[t]{2}{*}{$30 \mathrm{~mm}$} & 60 & 9 & 4.95 & 0.11 & 2.16 & -0.6 & 8.3 & -7.3 \\
\hline & 66 & 3 & 6.87 & 1.52 & 2.15 & -0.9 & 9.3 & -12.7 \\
\hline $100 \mathrm{MeV}$ & 34 & 15 & 4.48 & 0.12 & 1.83 & 0.0 & 9.3 & -3.3 \\
\hline $\begin{array}{l}49 \mathrm{~mm} \\
30 \mathrm{~mm}\end{array}$ & 45 & 4 & 6.80 & 1.22 & 1.86 & 1.0 & 12.5 & -10.8 \\
\hline
\end{tabular}

range, which has not been accurately measured by RGD. Since it directly uses LET, which explains the physical mechanism underlying the quenching effect, our method quantitatively compensates the quenching effect and can be applied to any combination of a proton beam and RGD, e.g., in combination with scanning methods. RGDs can be potentially used in proton postal measurement and in vivo dosimetry, and an efficient analysis of the observations of this study can lead to a more accurate dosimetry and estimation of error factors. However, especially for IMPT, LET may not be accurately calculated by a uniform index such as $\mathrm{R}_{\text {res}}$. Therefore, a method combining the measurements with MC 
calculations, such as the method used in this study, can be useful. Although LET-based correction methods using MC simulations are timeconsuming, labor-intensive, and require knowledge of the calculation code, it is expected that the TPS will be able to estimate LET more easily [37]. In addition, analytical LET calculations based on the pencil-beam algorithm and LET measurements using a sealed miniaturized Tissue Equivalent Proportional Counter have also been developed [38,39]. Based on the development of these methods for calculating LET and the results of this study, RGDs will prove to be useful for the postal audit of multicenter trial using scanning methods such as IMPT. In a report showing the LET dependence of other dosimeters, such as Gafchromic films and ionization chambers, the correction equation was calculated by a linear fitting $[6,40]$. Since the RGD contains more high atomic number materials than these dosimeters and exhibits a different measurement mechanism, the power law function may have provided a better fitting result. The dependence of the ionization chambers on LET has been demonstrated for beams with a large LET variation (e.g., carbon beams) [40]; clearly, a wider range of LET investigations is needed for RGDs. The cause of the decreased response of RGDs with increasing LET is not yet clear. In the literature, the decrease in luminescence efficiency with increasing LET is attributed to a decrease in the probability of electron-hole traps adjacent to ion pathways where ionization is dense, resulting in a decrease in the efficiency to form luminescent centers [41]. The decrease in response of RGDs with increasing LET in the present study is considered to follow a similar mechanism.

The LET-corrected RGD doses agreed well with the ionization chamber doses $(0.04 \pm 2.1 \%)$. The largest error, which was estimated to be approximately $5.3 \%$ for a $R_{\text {res }}$ of $3 \mathrm{~mm}$, was due to the dose or LET uncertainty caused by the positional uncertainty of the measurement. Rres-correction showed a large error of approximately $10 \%$ when the residual range was less than $2 \mathrm{~cm}$, but the error was significantly less $(0.7 \pm 2.3 \%)$ for residual range greater than $2 \mathrm{~cm}$. Further, because of the simplicity of the method, the $\mathrm{R}_{\text {res}}$-based correction was effective when LET was close to the calibration condition.

In Europe and the United States, TLDs have been used for postal audit of proton therapy $[13,42]$, however, the postal audit protocol for proton beam has not been established in Japan. The RGD are used for postal audits of various irradiation methods such as IMRT for their excellent dose characteristics in photon therapy [10-13]. In our previous study, the uncertainty in RGD's postal dosimetry for proton beam was estimated to be $3.6 \%$; among them, the uncertainty due to LET was expected to be $3.0 \%$ [20]. This uncertainty is reduced by the LET correction factor established in this study. In this study, the RGDs were calibrated and validated at LET of $3.3 \mathrm{keV} / \mu \mathrm{m}$ (reference condition: $200 \mathrm{MeV} / \mathrm{SOBP} 10$ $\mathrm{cm}$, SOBP center). Therefore, the RGD under-response has to be considered when calibrating using low LET photon beams.

The uncertainties in this study are primarily the positional uncertainty in both the ionization chamber and RGD measurements. The effective measurement point of the ionization chamber is assumed to be approximately $1 \mathrm{~mm}$ toward the source [43]; however, the effective measurement point of an RGD has not been investigated and verified. The position of the effective center of an RGD may be different from that of the ionization chamber because an RGD contains high atomic number materials. In this study, the uncertainty was estimated by assuming a positional deviation of $\pm 2 \mathrm{~mm}$ from the effective measurement point and depth scaling of the water-equivalent phantom. Moreover, the RGD has a sensitive area of $1 \mathrm{~mm}$ diameter, which limits the measurement because of the partial volume effect. In the case of particle dosimetry, the Bragg peak may cause steep dose gradients in the depth direction, which should be considered regardless of the type of detector used [8]. In addition, we calculated the LET in water in the MC calculation. In practice, however, the LET in the RGD may be different from the LET in the water when a high-atomic number material such as RGD is inserted. A detailed study of LET variation in the presence or absence of RGD is much needed. Although these uncertainties are limitations of the study, the correction equation based on the LET established in this study shows a very good agreement to ionization chamber dose with $0.04 \pm 2.1 \%$, thus verifying the usefulness of RGD.

\section{Conclusions}

We studied the LET-dependent quenching effect of RGD using therapeutic proton beams. The results of this study suggest that the LETdependence of RGD can be corrected using a correction formula based on a power function of LET. This correction method enables precise dosimetric verification near the end of proton range and low-energy proton beam. Although the RGD response to the systems using ridge filters and the scanning needs to be verified, LET-based correction explains the physical mechanism underlying the quenching effect, and our method may be commonly used for all irradiation methods. These results will be useful in establishing a postal audit system and in-vivo dosimetry using RGD for proton beams.

\section{Acknowledgment}

This work was supported by JSPS KAKENHI grant number JP19K17181.

\section{References}

[1] Kanai T, Kawachi K, Kumamoto Y, Ogawa H, Yamada T, Matsuzawa H, Inada T. Spot scanning system for proton radiotherapy. Med Phys 1980;7:365-9.

[2] Smith A, Gillin M, Bues M, Zhu XR, Suzuki K, Mohan R, Woo S, Lee A, Komaki R, Cox J, Hiramoto K, Akiyama H, Ishida T, Sasaki T, Matsuda K. The M. D. Anderson proton therapy system: The M. D. Anderson proton therapy system. Med Phys 2009;36:4068-83.

[3] Gillin MT, Sahoo N, Bues M, Ciangaru G, Sawakuchi G, Poenisch F, Arjomandy B, Martin C, Titt U, Suzuki K, Smith AR, Zhu XR. Commissioning of the discrete spot scanning proton beam delivery system at the University of Texas M.D. Anderson Cancer Center, Proton Therapy Center, Houston: Commissioning of a discrete spot scanning proton beam. Med Phys 2010;37:154-63.

[4] Mirandola A, Molinelli S, Vilches Freixas G, Mairani A, Gallio E, Panizza D, et al. Dosimetric commissioning and quality assurance of scanned ion beams at the Italian National Center for Oncological Hadrontherapy: commissioning and QA of actively scanned particle beams. Med Phys 2015;42:5287-300.

[5] Molinelli S, Russo S, Magro G, Maestri D, Mairani A, Mastella E, et al. Impact of TPS calculation algorithms on dose delivered to the patient in proton therapy treatments. Phys Med Biol 2019;64:075016. https://doi.org/10.1088/1361-6560/ ab0a4d.

[6] Anderson SE, Grams MP, Wan Chan Tseung H, Furutani KM, Beltran CJ. A linear relationship for the LET-dependence of Gafchromic EBT3 film in spot-scanning proton therapy. Phys Med Biol 2019;64:055015. DOI:10.1088/1361-6560/ab0114.

[7] Kohno R, Hotta K, Matsubara K, Nishioka S, Matsuura T, Kawashima M. In vivo proton dosimetry using a MOSFET detector in an anthropomorphic phantom with tissue inhomogeneity. J Appl Clin Med Phys 2012;13:159-67. https://doi.org/ 10.1120/jacmp.v13i2.3699.

[8] Kry SF, Alvarez P, Cygler JE, DeWerd LA, Howell RM, Meeks S, O’Daniel J, Reft C, Sawakuchi G, Yukihara EG, Mihailidis D. AAPM TG 191: clinical use of luminescent dosimeters: TLDs and OSLDs. Med Phys 2020;47. https://doi.org/10.1002/mp. v47.210.1002/mp.13839.

[9] Rah JE, Oh DH, Kim JW, Kim DH, Suh TS, Ji YH, et al. Feasibility study of glass dosimeter for in vivo measurement: Dosimetric characterization and clinical application in proton beams. Int J Radiat Oncol Biol Phys 2012;84:251-6. https:// doi.org/10.1016/j.ijrobp.2012.03.054.

[10] Marsolat F, De Marzi L, Patriarca A, Nauraye C, Moignier C, Pomorski M, Moignau F, Heinrich S, Tromson D, Mazal A. Dosimetric characteristics of four PTW microDiamond detectors in high-energy proton beams. Phys Med Biol 2016; 61(17):6413-29. https://doi.org/10.1088/0031-9155/61/17/6413.

[11] Zullo JR, Kudchadker RJ, Zhu XR, Sahoo N, Gillin MT. LiF TLD-100 as a dosimeter in high energy proton beam therapy—can it yield accurate results? Med Dosim 2010;35:63-6. https://doi.org/10.1016/j.meddos.2009.03.001.

[12] Reft CS. The energy dependence and dose response of a commercial optically stimulated luminescent detector for kilovoltage photon, megavoltage photon, and electron, proton, and carbon beams: energy dependence of optically stimulated luminescent detector. Med Phys 2009;36:1690-9. https://doi.org/10.1118/ 1.3097283.

[13] Taylor PA, Kry SF, Alvarez P, Keith T, Lujano C, Hernandez N, Followill DS. Results from the imaging and radiation oncology core houston's anthropomorphic phantoms used for proton therapy clinical trial credentialing. Int J Radiat Oncol Biol Phys 2016;95:242-8. https://doi.org/10.1016/j.ijrobp.2016.01.061.

[14] Rossomme S, Denis JM, Souris K, Delor A, Bartier F, Dumont D, Vynckier S, Palmans H. LET dependence of the response of a PTW-60019 microDiamond detector in a $62 \mathrm{MeV}$ proton beam. Phys Med 2016;32:1135-8. https://doi.org/ 10.1016/j.ejmp.2016.08.017. 
[15] Rossomme S, Marinelli M, Verona-Rinati G, Romano F, Cirrone PAG, Kacperek A, Vynckier S, Palmans H. Response of synthetic diamond detectors in proton, carbon, and oxygen ion beams. Med Phys 2017;44:5445-9. https://doi.org/10.1002/ mp. 12473.

[16] Mizuno H, Kanai T, Kusano Y, Ko S, Ono M, Fukumura A, Abe K, Nishizawa K, Shimbo M, Sakata S, Ishikura S, Ikeda H. Feasibility study of glass dosimeter postal dosimetry audit of high-energy radiotherapy photon beams. Radiother Oncol 2008; 86:258-63. https://doi.org/10.1016/j.radonc.2007.10.024.

[17] Mizuno H, Fukumura A, Fukahori M, Sakata S, Yamashita W, Takase N, Yajima K, Katayose T, Abe-Sakama K, Kusano Y, Shimbo M, Kanai T. Application of a radiophotoluminescent glass dosimeter to nonreference condition dosimetry in the postal dose audit system: dose audit using glass dosimeter to nonreference condition. Med Phys 2014;41:112104. https://doi.org/10.1118/1.4898596.

[18] Okamoto H, Minemura T, Nakamura M, Mizuno H, Tohyama N, Nishio T, et al. Establishment of postal audit system in intensity-modulated radiotherapy by radiophotoluminescent glass dosimeters and a radiochromic film. Phys Med 2018; 48:119-26. https://doi.org/10.1016/j.ejmp.2018.03.013.

[19] Kamomae T, Shimizu H, Nakaya T, Okudaira K, Aoyama T, Oguchi H, et al. Threedimensional printer-generated patient-specific phantom for artificial in vivo dosimetry in radiotherapy quality assurance. Phys Med 2017;44:205-11. https:// doi.org/10.1016/j.ejmp.2017.10.005.

[20] Chang W, Koba Y, Katayose T, Yasui K, Omachi C, Hariu M, et al. Correction of stopping power and LET quenching for radiophotoluminescent glass dosimetry in a therapeutic proton beam. Phys Med Biol 2017;62. DOI:10.1088/1361-6560/ aa9155.

[21] Rah J-E, Oh DH, Shin D, Lee SB, Kim TH, Kim J-Y, et al. Feasibility study of glass dosimeter for postal dose intercomparison of high-energy proton therapy beams. Radiat Meas 2013;59:66-72. https://doi.org/10.1016/j.radmeas.2013.10.004.

[22] Araki F, Ohno T. The response of a radiophotoluminescent glass dosimeter in megavoltage photon and electron beams: the response of a radiophotoluminescent glass dosimeter. Med Phys 2014;41:122102. https://doi.org/10.1118/1.4901639.

[23] Toshito T, Omachi C, Kibe Y, Sugai H, Hayashi K, Shibata H, et al. A proton therapy system in Nagoya Proton Therapy Center. Australas Phys Eng Sci Med 2016;39: 645-54. https://doi.org/10.1007/s13246-016-0456-8.

[24] Iwata H, Ishikawa H, Takagi M, Okimoto T, Murayama S, Akimoto T, et al. Longterm outcomes of proton therapy for prostate cancer in Japan: a multi-institutional survey of the Japanese Radiation Oncology Study Group. Cancer Med 2018;7: 677-89. https://doi.org/10.1002/cam4.1350.

[25] Nakajima K, Iwata H, Ogino H, Hattori Y, Hashimoto S, Nakanishi M, Toshito T, Umemoto Y, Iwatsuki S, Shibamoto Y, Mizoe J-E. Acute toxicity of image-guided hypofractionated proton therapy for localized prostate cancer. Int J Clin Oncol 2018;23:353-60. https://doi.org/10.1007/s10147-017-1209-8.

[26] Nakajima K, Iwata H, Ogino H, Hattori Y, Hashimoto S, Toshito T, et al. Clinical outcomes of image-guided proton therapy for histologically confirmed stage I nonsmall cell lung cancer. Radiat Oncol 2018;13. https://doi.org/10.1186/s13014018-1144-5.

[27] Yasui K, Shimomura A, Toshito T, Tanaka K, Ueki K, Muramatsu R, Katsurada M, Hayashi N, Ogino H. A quality assurance for respiratory gated proton irradiation with range modulation wheel. J Appl Clin Med Phys 2019;20:258-64. https://doi. org/10.1002/acm2.12526.
[28] Kadoya N, Shimomura K, Kitou S, Shiota Y, Fujita Y, Dobashi S, et al. Dosimetric properties of radiophotoluminescent glass detector in low-energy photon beams. Med Phys 2012;39:5910-6. https://doi.org/10.1118/1.4747261.

[29] Rah J-E, Oh DH, Shin D, Kim D-H, Ji YH, Kim JW, et al. Dosimetric evaluation of a glass dosimeter for proton beam measurements. Appl Radiat Isot 2012;70: 1616-23. https://doi.org/10.1016/j.apradiso.2012.04.007.

[30] Araki F, Kubo HD. Comparison of high-energy photon and electron dosimetry for various dosimetry protocols. Med Phys 2002;29:857-68. https://doi.org/10.1118/ 1.1470208.

[31] Araki F, Ikegami T, Ishidoya T, Kubo HD. Measurements of Gamma-Knife helmet output factors using a radiophotoluminescent glass rod dosimeter and a diode detector. Med Phys 2003;30:1976-81. https://doi.org/10.1118/1.1587451.

[32] JSMP (Japan Society of Medical Physics). Standard dosimetry of absorbed dose to water in external beam radiotherapy (in Japanese). Tokyo, Tsusho-SangyoKenkyusha; 2012.

[33] Vynckier S, Bonnett DE, Jones DTL. Supplement to the code of practice for clinical proton dosimetry. Radiother Oncol 1994;32:174-9. https://doi.org/10.1016/ 0167-8140(94)90104-X.

[34] IAEA TRS398. Absorbed Dose Determination in External Beam Radiotherapy. Vieena, IAEA; 2000. DOI:10.1097/00004032-200111000-00017.

[35] Agostinelli S, Allison J, Amako K, Apostolakis J, Araujo H, Arce P, et al. Geant4-a simulation toolkit. Nucl Instrum Methods Phys Res, Sect A 2003;506:250-303. https://doi.org/10.1016/S0168-9002(03)01368-8.

[36] Allison J, Amako K, Apostolakis J, Arce P, Asai M, Aso T, et al. Recent developments in Geant4. Nucl Instrum Methods Phys Res, Sect A 2016;835: 186-225. https://doi.org/10.1016/j.nima.2016.06.125.

[37] Wagenaar D, Tran LT, Meijers A, Marmitt GG, Souris K, Bolst D, et al. Validation of linear energy transfer computed in a Monte Carlo dose engine of a commercial treatment planning system. Phys Med Biol 2020;65. DOI:10.1088/1361-6560/ ab5e97.

[38] Hirayama S, Matsuura T, Ueda H, Fujii Y, Fujii T, Takao S, et al. An analytical doseaveraged LET calculation algorithm considering the off-axis LET enhancement by secondary protons for spot-scanning proton therapy. Med Phys 2018;45:3404-16. https://doi.org/10.1002/mp.12991.

[39] Conte V, Bianchi A, Selva A, Petringa G, Cirrone GAP, Parisi A, Vanhavere F, Colautti P. Microdosimetry at the CATANA $62 \mathrm{MeV}$ proton beam with a sealed miniaturized TEPC. Physica Med 2019;64:114-22. https://doi.org/10.1016/j. ejmp.2019.06.011.

[40] Mirandola A, Magro G, Maestri D, Mairani A, Mastella E, Molinelli S, et al. Determination of ion recombination and polarity effect correction factors for a plane-parallel ionization Bragg peak chamber under proton and carbon ion pencil beams. Phys Med Biol 2019;64. DOI:10.1088/1361-6560/ab0db4.

[41] Yasuda H, Fujitaka K. Responses of TLD-Mg2SiO4:Tb and radiophotoluminescent glass to heavy charged particles and space radiation. Radiat Prot Dosim 2000;87: 115-9. https://doi.org/10.1093/oxfordjournals.rpd.a032983.

[42] Kunst J, Kopeć R, Kukołowicz P, Mojżeszek N, Sadowski B, Stolarczyk L, et al. Mailed dosimetric audit of therapeutic proton beams using thermoluminescence MTS-N (LiF:Mg,Ti) powder - first results. Radiat Meas 2017;106:312-4. https:// doi.org/10.1016/j.radmeas.2017.03.031.

[43] Yasui K, Toshito T, Omachi C, Hayashi K, Kinou H, Katsurada M, et al. Dosimetric verification of IMPT using a commercial heterogeneous phantom. J Appl Clin Med Phys 2019;20:114-20. https://doi.org/10.1002/acm2.12535. 